\title{
Analysis of Some Online Questions with High Frequency about Dental Treatment in Korea
}

\author{
A-Reum Kang, Ye-Eun Go, Ka-Eun Kim, Min-Joo Kim, Seon-Jeong Kim, and SooJeong Hwang ${ }^{\dagger}$ \\ Department of Dental Hygiene, College of Medical Science, Konyang University, Daejeon 35365, Korea
}

\begin{abstract}
Background: The Internet has advantages in terms of accessibility and amount of information, and the search for health information over the Internet is increasing exponentially. The purpose of this study is to analyze the information generated about some dental treatment on the internet by year. Methods: Naver Knowledge (Jisikln in Korean) which is an interactive search service was selected as the first search site in Korea. Scaling, wisdom tooth extraction, and endodontic treatment that can be paid by Korean health insurance were selected. Finally, 4,729 questions about scaling, 23,963 wisdom teeth extraction questions and 17,733 endodontic treatment questions were extracted. The question contents, the information about the questioner and the answerer, and an error of answers were investigated. Frequency analysis was used and chi-square test was used if necessary.

Results: The most frequently asked questions were discomfort and dissatisfaction after the treatment. The need for treatment was the second in questions of the wisdom tooth extraction and endodontic treatment, but the health insurance benefit was the second in dental scaling. Most of the questioners didn't disclose personal information. The public answered the most in 2013 2014, but the highest percentage of the respondents was experts in 2017. Responses were mostly personal experience, but showed a tendency to decrease with years, and professional knowledge showed an increasing tendency. The error of the answer has also gradually decreased.

Conclusion: Questions about dental care over the Internet are increasing exponentially, experts are responding increasingly, and errors in answers are decreasing. Nevertheless, it is necessary to pay attention to the related expert group to prevent misinformation.
\end{abstract}

Key Words: Dental care, Internet, Korea, Online social networking

\section{Introduction}

Demand for online health information has increased rapidly due to changes in health awareness owing to the rise in internet usage, expansion of medical supplies, increase in medical knowledge and rights, and improvements in living standards ${ }^{1}$. The use of social media to resolve simple daily-life medical problems has become common mostly because of its convenience. It has been shown that online health information is sought mostly by those who start identifying unusual symptoms through self-diagnosis or those who are healthy but are looking to maintain their health ${ }^{2}$. However, online health information has issues such as accessibility, poor quality, difficulties in finding and interpreting the information, and risk of the information being over-used. In particular, although a great number of websites provide health information, the quality of their information is still questionable ${ }^{3)}$.

Social media is a service or website that facilitates the expression of social relationships between people who share interests or real-world relationships in a virtual community ${ }^{4}$. While there are many online search engines in Korea, Naver Knowledge (JisikIn in Korean) is the top ranked one with more than $80 \%$ market share, followed by Daum Knowledge and Nate Knowledge. Naver Knowledge received 11,532 new questions, and generated 12,427 answers on a single day (November 9, 2015) ${ }^{5}$. It provides a platform for interactions amongst general public, or 
between general public and experts through questions and answers $^{4)}$. It has the advantage of providing specific information concisely in accordance with the inquirer's intention and other users are more likely to obtain the answers needed since the inquirer can select the most reliable answer from among many other answers ${ }^{4)}$.

Several studies have been conducted on dental treatment ${ }^{6}$ or demand for information on dental hygienists ${ }^{7,8)}$ using Naver knowledge; but it has been a while since these studies were conducted, although the rate of accumulation of data on the internet has increased exponentially every year. It is, therefore, necessary to examine whether the internet has provided the patient with correct dental treatment information. This study aims at analyzing the dental care related questions and answers generated by Naver Knowledge between 2013 and 2017, to investigate errors in the contents, as well as the changing trends in these errors year wise.

\section{Materials and Methods}

\section{Object and data collection}

The subject matter comprised questions posted on Naver Knowledge about dental treatment covered by health insurance. Treatments were classified into periodontal, restorative, and surgical treatments. Additionally, treatments frequently asked on Naver Knowledge using certain keywords, and that were rarely covered by insurance but frequently performed according to previous research ${ }^{9-12)}$, were selected. Five-year data collected from January 1, 2013 to December 31, 2017 on Naver Knowledge was studied. The questions and answers were classified using keywords like 'scaling,' 'wisdom tooth extraction,' and 'root canal treatment,' frequently used by the general public from April 1, 2018 to July 10, 2018. The words scaling and neurotherapy are also used in fields other than dentistry. Hence, based on data that the word scaling was used for dogs, cats, dermatology, skin, scalp, resolution, and monitors; moreover, the word "endodontic treatment" was used for lower back, vertebrae, and brain, and these were excluded after researchers had adequate discussions and literature review was concluded. The questions or answers were searched if a certain term was included, and only the words found in the questions were extracted for the final analysis. When two or more terms were searched in the same question, researchers discussed the intent of the questions and decided how to categorize them. Fig. 1 illustrates the number of each term retrieved and classified year-wise.

\section{Analysis method}

This study was designed based on a study by Kim and $\mathrm{Lim}^{13)}$. A month from each year was randomly selected for the first preliminary survey, based on which, the classification criteria were set for the questions, responders, and answers. The answers were classified into three categories: expert knowledge; personal knowledge and experience; and dental visit recommendation. Accuracy of these answers were determined based on references and related knowledge using 14 textbooks in dental hygiene, including periodontology, oral prophylaxis, oral and maxillofacial surgery, and dental restoration. Questions were categorized considering cost, insurance, necessity of treatment, pain during treatment, pre- and postoperative care, combination with other treatments, discomfort and complaints after treatment, treatment outcomes, treatment plan, care after

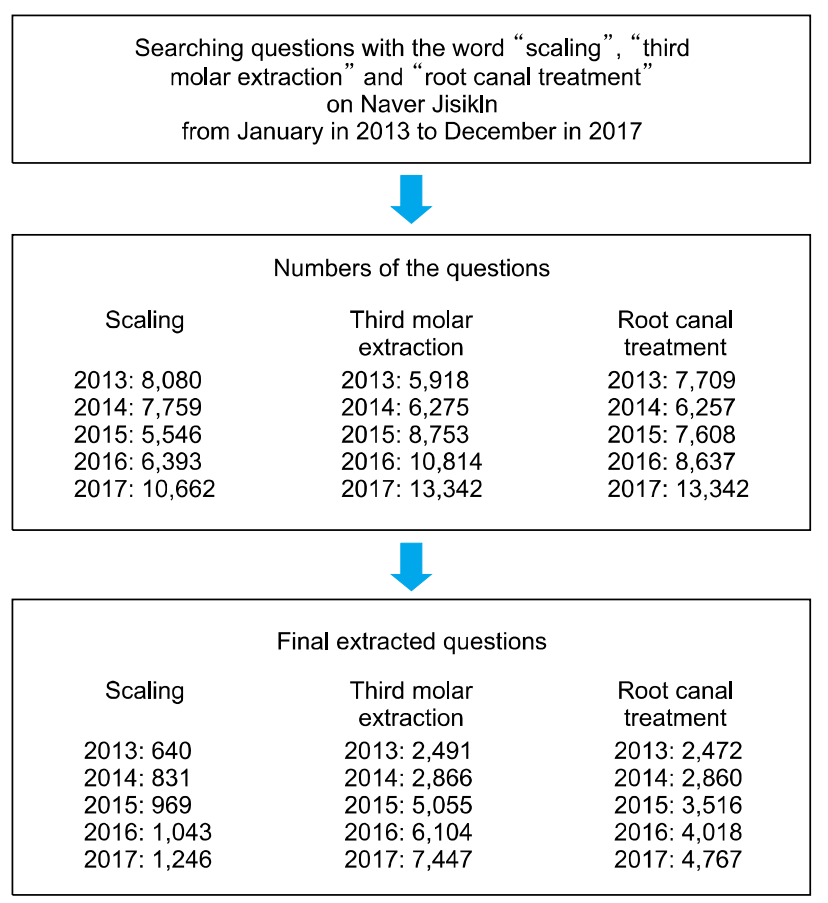

Fig. 1. Flow chart of extracting the data in Naver Knowledge. 
treatment, discomfort after treatment discontinuation, and retreatment. Information on inquirers and responders' characteristics were collected such as responders' gender and age groups, and information pertaining to whether the responders were anonymous, general public, or experts was assessed. Only dentists and dental hygienists were considered as professional responders.

Both frequency and multiple response analysis were performed using the IBM SPSS ver. 20.0 (IBM Corp., Armonk, NY, USA) program. Chi-square tests were conducted for statistical analysis.

\section{Results}

1. Questions on scaling, wisdom tooth extraction, and endodontic treatment in Naver Knowledge from 2013 to 2017

During 2013 to 2017, the most frequently asked questions on Naver Knowledge regarding scaling were about discomfort and complaints after the procedure, followed by dental coverage, necessity of scaling, cost, discomfort during the procedure, treatment outcomes, pre and post-care after scaling, and possibility of combination with other dental treatments (Fig. 2). The number of questions pertaining to insurance coverage and benefits of scaling was highest in 2013, but became less frequent afterwards, as compared to questions on discomfort and complaints after the procedure.

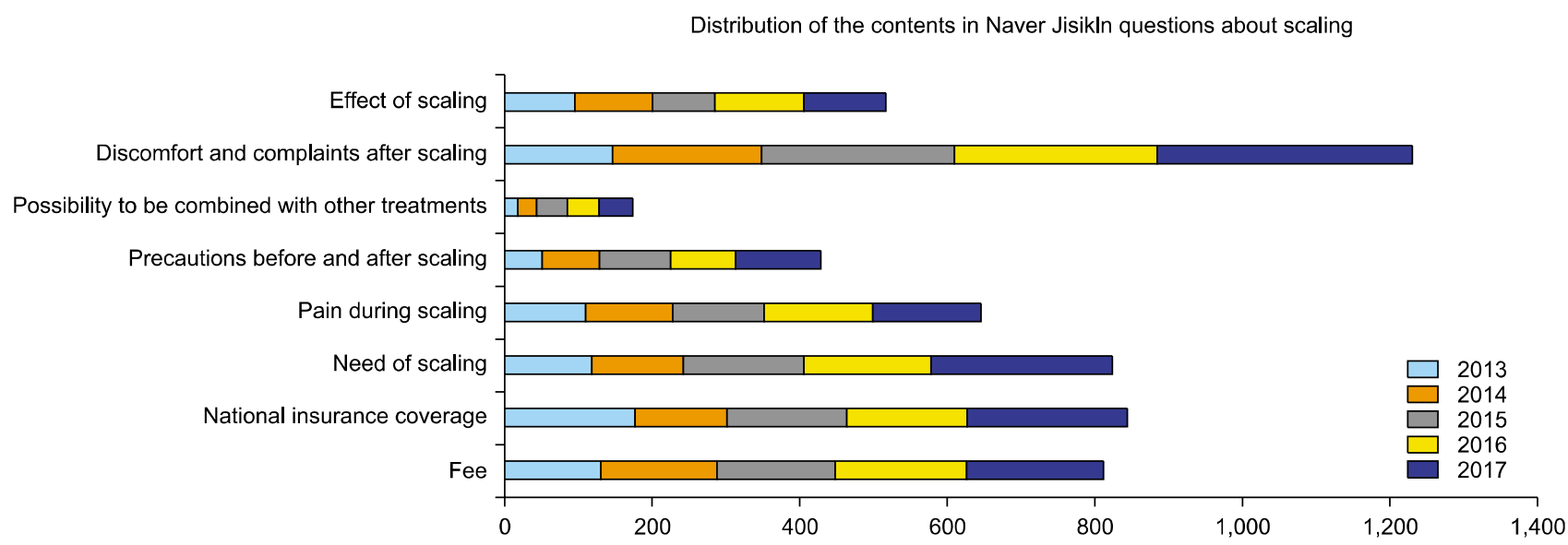

Fig. 2. Distribution of the contents in Naver Knowledge questions about scaling.

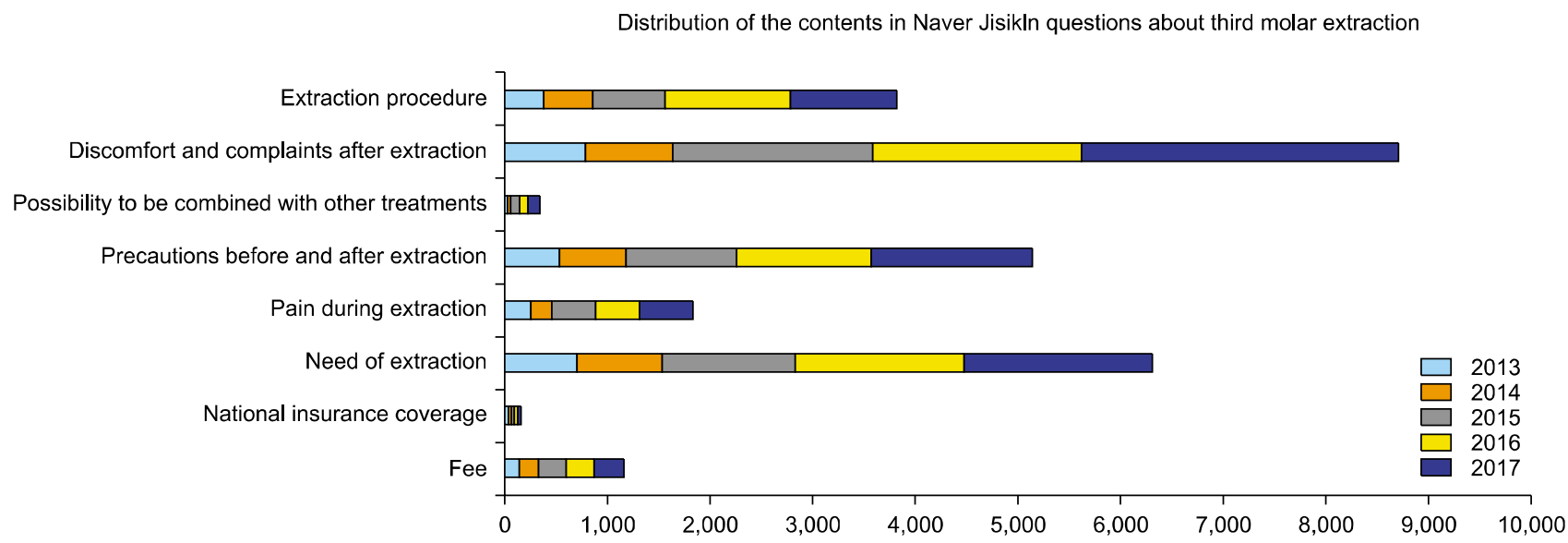

Fig. 3. Distribution of the contents in Naver Knowledge questions about third molar extraction. 
The frequency of questions about discomfort and dissatisfaction after wisdom tooth extraction was the highest, followed by necessity of wisdom teeth extraction, pre- and post-care after extraction, treatment or surgery procedure, pain, cost, possibility of other dental treatment parallelly, and benefits and coverage of health insurance (Fig. 3). Significant yearly changes in frequency of topics regarding wisdom tooth extraction were not observed. For endodontic treatment, the questions on discomfort after the procedure showed the highest frequency, followed by necessity of endodontic treatment, treatment or surgical procedure, pain, pre- and post-care, follow-up treatments, discomfort and retreatment due to discontinuation, possibility of combination with other dental treatments, health insurance benefits and coverage (Fig. 4). These frequencies changed every year, but no consistent trend was observed.

\section{Personal information of inquirers and responders} on scaling, wisdom tooth extraction, and endodontic treatment in Naver Knowledge during 2013 2017 Most of the questioners did not disclose personal information such as gender, hence the frequency of nondisclosure increased slightly over time (Table 1).

Unlike the responders, more than half of the inquirers disclosed their personal information (Table 2). The questions with multiple answerers were considered as duplicate responses, and the percentage of the number of questions to one of the answers was calculated. The rate of pro-

Distribution of the contents in Naver Jisikln questions about root canal treatment

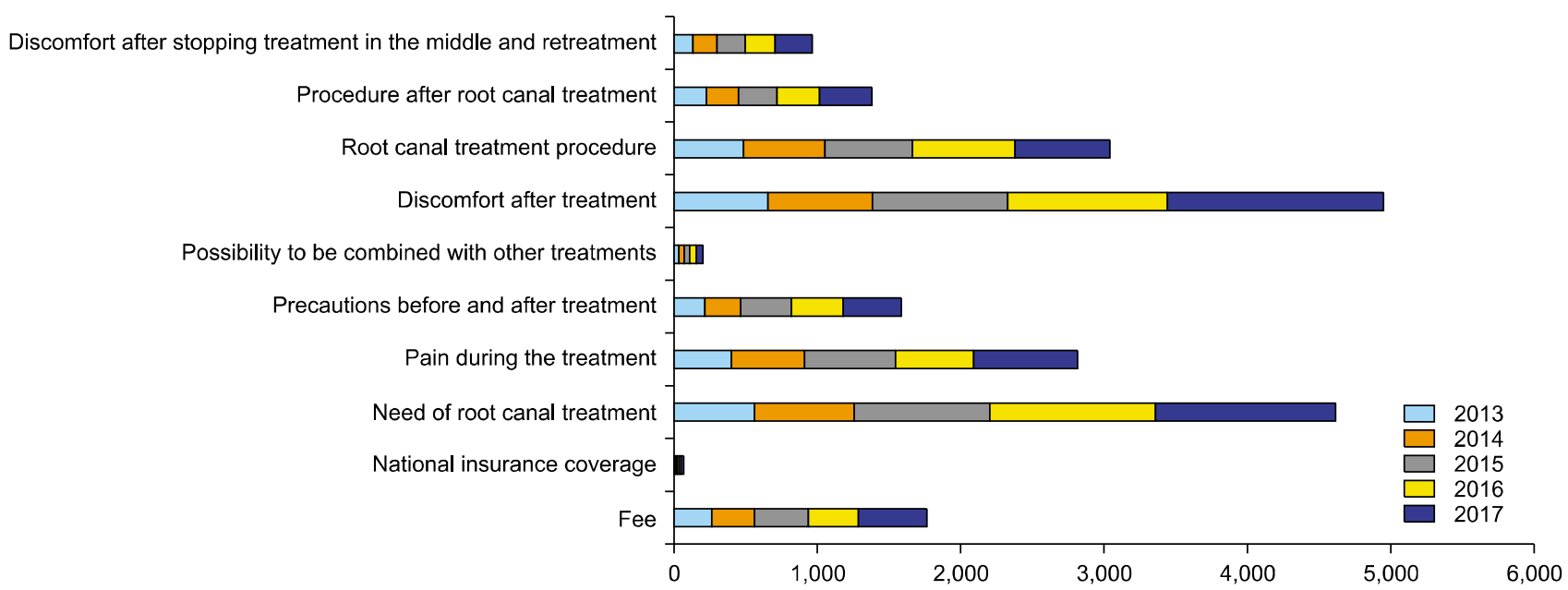

Fig. 4. Distribution of the contents in Naver Knowledge questions about endodontic treatment.

Table 1. Distribution of Sex in the Questioners according to Scaling, Third Molar Extraction, and Endodontic Treatment

\begin{tabular}{|c|c|c|c|c|c|c|c|}
\hline Category & Sex & 2013 & 2014 & 2015 & 2016 & 2017 & p-value \\
\hline \multirow[t]{3}{*}{ Scaling } & Unknown & $605(94.5)$ & $793(95.4)$ & $928(95.8)$ & $999(95.8)$ & $1,202(96.5)$ & 0.100 \\
\hline & Male & $21(3.3)$ & $11(1.3)$ & $22(2.3)$ & $22(2.1)$ & $23(1.8)$ & \\
\hline & Female & $14(2.2)$ & $27(3.2)$ & $19(2.0)$ & $22(2.1)$ & $21(1.7)$ & \\
\hline \multirow[t]{3}{*}{ Third molar extraction } & Unknown & $2,416(97.0)$ & $2,793(97.4)$ & $4,930(97.5)$ & $5,986(98.1)$ & $7,326(98.4)$ & $<0.001$ \\
\hline & Male & $39(1.6)$ & $39(1.4)$ & $59(1.2)$ & $70(1.1)$ & $77(1.0)$ & \\
\hline & Female & $36(1.4)$ & $35(1.2)$ & $66(1.3)$ & $48(0.8)$ & $44(0.6)$ & \\
\hline \multirow[t]{3}{*}{ Endodontic treatment } & Unknown & $2,389(96.6)$ & $2,797(96.7)$ & 3,409 (97.0) & $3,923(97.6)$ & $4,662(97.8)$ & 0.010 \\
\hline & Male & $25(1.0)$ & $29(1.0)$ & $32(0.9)$ & $32(0.8)$ & $46(1.0)$ & \\
\hline & Female & $58(2.3)$ & $64(2.2)$ & $75(2.1)$ & $63(1.6)$ & $59(1.2)$ & \\
\hline p-value & & $<0.001$ & 0.001 & 0.002 & $<0.001$ & $<0.001$ & \\
\hline
\end{tabular}

Values are presented as $\mathrm{n}(\%)$.

$\mathrm{p}$-values were obtained through $\chi^{2}$-test. 
Table 2. Distribution of Respondents according to Scaling, Third Molar Extraction, and Endodontic Treatment

\begin{tabular}{llcccccc}
\hline \multicolumn{1}{c}{ Variable } & \multicolumn{1}{c}{ Respondent } & \multicolumn{1}{c}{2013} & \multicolumn{1}{c}{2014} & \multicolumn{1}{c}{2015} & 2016 & 2017 & p-value \\
\hline Scaling & Private & $274(42.8)$ & $409(33.0)$ & $354(36.5)$ & $422(40.5)$ & $526(42.2)$ & $<0.001$ \\
& Professionals & $224(35.0)$ & $250(30.1)$ & $471(48.6)$ & $418(40.1)$ & $566(45.4)$ & \\
& Non-professionals & $511(79.8)$ & $534(64.3)$ & $411(42.4)$ & $500(47.9)$ & $534(42.9)$ & \\
Third molar extraction & Private & $863(94.6)$ & $1,172(40.9)$ & $1,299(25.7)$ & $1,547(25.3)$ & $2,539(34.1)$ & $<0.001$ \\
& Professionals & $1,415(56.8)$ & $1,333(46.5)$ & $3,669(72.6)$ & $3,615(59.2)$ & $4,413(59.3)$ & \\
Endodontic treatment & Non-professionals & $1,702(68.3)$ & $1,657(57.8)$ & $1,672(33.1)$ & $2,175(35.6)$ & $2,357(31.7)$ & \\
& Private & $980(39.4)$ & $1,017(35.6)$ & $1,112(31.6)$ & $1,497(37.3)$ & $1,900(39.9)$ & $<0.001$ \\
& Professionals & $840(34.0)$ & $909(31.8)$ & $1,853(52.7)$ & $1,481(36.9)$ & $2,037(42.7)$ & \\
p-value & Non-professionals & $1,640(66.3)$ & $1,768(61.8)$ & $1,394(39.7)$ & $1,872(46.6)$ & $1,670(35.0)$ & $<0.001$ \\
\hline
\end{tabular}

Values are presented as $\mathrm{n}(\%)$.

p-values were obtained through $\chi^{2}$-test.

Table 3. Distribution of Answer Contents according to Scaling, Third Molar Extraction, and Endodontic Treatment

\begin{tabular}{|c|c|c|c|c|c|c|c|}
\hline Variable & Answer content & 2013 & 2014 & 2015 & 2016 & 2017 & p-value \\
\hline \multirow[t]{3}{*}{ Scaling } & Professional knowledge & $225(35.2)$ & $246(29.6)$ & $465(48.0)$ & $412(39.5)$ & $554(44.5)$ & $<0.001$ \\
\hline & Personal experience & $742(115.9)$ & $921(110.8)$ & 747 (77.1) & 907 (86.9) & $1,026(82.4)$ & \\
\hline & Dental clinic visit & $42(6.6)$ & $26(3.1)$ & $24(2.5)$ & $21(2.3)$ & $46(3.7)$ & \\
\hline \multirow[t]{3}{*}{ Third molar extraction } & Professional knowledge & $1,400(56.2)$ & $1,311(45.7)$ & $3,592(71.1)$ & $3,536(57.9)$ & $4,300(57.7)$ & $<0.001$ \\
\hline & Personal experience & $2,470(99.2)$ & $2,724(95.1)$ & $2,815(55.7)$ & $3,526(57.8)$ & $4,591(61.7)$ & \\
\hline & Dental clinic visit & $110(4.4)$ & $127(4.4)$ & $233(4.6)$ & $275(4.5)$ & $418(5.6)$ & \\
\hline \multirow[t]{3}{*}{ Endodontic treatment } & Professional knowledge & $828(34.3)$ & $900(31.5)$ & $1,805(51.3)$ & $1,456(36.2)$ & $2,003(42.0)$ & $<0.001$ \\
\hline & Personal experience & $2,525(102.1)$ & 2,691 (94.1) & $2,286(65.0)$ & $3,212(79.9)$ & $3,247(68.1)$ & \\
\hline & Dental clinic visit & $107(4.3)$ & $103(3.6)$ & $268(7.6)$ & $182(4.5)$ & $357(7.5)$ & \\
\hline p-value & & $<0.001$ & $<0.001$ & $<0.001$ & $<0.001$ & $<0.001$ & \\
\hline
\end{tabular}

Values are presented as $\mathrm{n}(\%)$.

p-values were obtained through $\chi^{2}$-test.

Table 4. Distribution of Answer Errors according to Each Treatment

\begin{tabular}{lccrrrr}
\hline \multicolumn{1}{c}{ Variable } & \multicolumn{1}{c}{2013} & \multicolumn{1}{c}{2014} & 2015 & 2016 & 2017 & $\mathrm{p}$-value \\
\hline Scaling & $100(15.7)$ & $104(12.5)$ & $74(7.6)$ & $62(5.9)$ & $73(5.9)$ & $<0.001$ \\
Third molar extraction & $294(11.8)$ & $225(7.9)$ & $240(4.7)$ & $231(3.8)$ & $553(7.4)$ & \\
Endodontic treatment & $296(12.0)$ & $265(9.3)$ & $248(7.1)$ & $247(6.2)$ & $258(5.3)$ & \\
\hline
\end{tabular}

Values are presented as $\mathrm{n}(\%)$.

p-values were obtained through $\chi^{2}$-test.

fessional responses increased from 2013 to 2015, decreased in 2016, and increased slightly in 2017.

\section{Classification of answers on scaling, wisdom tooth extraction, and endodontic treatment in Naver Knowledge in 2013 2017}

The questions with multiple responders were considered as duplicate responses (Table 3). Overall, personal experience accounted for the largest percentage of answers, as against 2015, when only wisdom tooth extraction had shown more professional responses than answers with personal experience. However, the proportion of responses with personal experience tended to decrease continuously every year. 
4. Accuracy and errors in answers on scaling, wisdom tooth extraction, and endodontic treatment in Naver Knowledge during 2013 2017

As shown in Table 4, the rate of errors in answers on scaling and endodontic treatment have continuously decreased. However, the rate of errors in answers on wisdom tooth extraction decreased until 2016 and then increased in 2017.

\section{Discussion}

The internet provides knowledge, information, communication, and collaboration, and has certain advantages in terms of accessibility and the amount of information compared to traditional sources, such as newspapers, television, or books ${ }^{14}$. From patients' points of view, the internet has positive effects on understanding diseases and improving quality of care and treatment outcomes ${ }^{15)}$. As the amount of information on diseases that patients could obtain on the internet increased, they became smarter consumers and were empowered, resulting in the growth of patient-centered care ${ }^{16)}$. Previous studies have found that using the internet to search for health related information tends to increase with the increase in the number of people who perceive use of health information as beneficial for them; and as more people observe unusual symptoms and diseases, and recognize the usefulness of the internet for promoting healthy behavior, awareness on prevention increases in their communities ${ }^{177}$.

Meanwhile, a lot of doctors are worried about the negative aspects of online health information. Some patients believe online health information blindly and ignore doctors' medical knowledge ${ }^{18)}$ and such information results in a rise in medical expenses, unnecessary hospital visits, and longer treatment time ${ }^{15)}$. It is considered that the basis for doctors' negative evaluation is inaccuracy or irrelevance of online health information. A previous study concluded that while it is true that the internet has enabled quick and easy access to information and contributed to the development of healthcare, concerns about the side effects of inaccurate information have increased and it would take a lot of time and effort to rectify information once it has been disseminated online ${ }^{19)}$. Therefore, even if the internet serves as an open source of health information, the accuracy or relevance of this information needs to be evaluated by experts.

Online dental information was collected from 2013 to 2017 using Naver Knowledge that provided two-sided interactions and had the largest market share in Korea. Given that a previous study used 4,212 data items from 2002 to $2012^{6}$, this study used data since 2013 . While the previous study collected about 4,000 data-points on oral health for 11 years, since 2013, thousands of data-points were searched annually with only one treatment name such as scaling; thus indicating that the use of the internet to search for oral health related information had increased drastically. In all three categories of treatments, questions on discomfort after procedures was most frequently asked, followed by necessity of treatment. This showed a significant difference from the previous study, that concluded that questions about symptoms (33.1\%) and treatment or surgery procedure $(22.6 \%)$ were most frequently asked ${ }^{6}$. The three stages of behavior based on health status include maintenance and promotion of wellness, self-recognition of symptoms, and search of medical services and disease treatment ${ }^{20)}$. While previous studies interpreted internet usage as second stage behavior that involves a search for medical service, in this study, such usage is interpreted as third stage behavior or disease treatment. This might be caused by the data collection methodology that used the names of procedures rather than the names of diseases.

The trend of decreasing rate of responses that integrated personal experiences was positive; but the expert response rate being less than $60 \%$ suggests the necessity to bring about improvements in virtual space that the experts can use to respond easily. It is also necessary to find professional manpower for relevant organizations to regulate and deliver accurate information in the virtual world that is not stationary and where institutional controls, such as law, are limited. For questions regarding scaling, that are mainly done by dental hygienists, the rates of answers that were written by experts and included professional knowledge were less than that of wisdom tooth extraction. Therefore, it is recommended that the Korean Dental Hygienists' Association or other relevant associations build enough manpower to answer questions regarding dental 
hygienists or allocate work among themselves.

In a study by Jeong et al. ${ }^{1)}$, one of the study participants pointed out that the risks of finding inaccurate or false information owing to the nature of the internet was very high and it was unclear whether the source of such information was responsible for providing misleading information. Kim et al. ${ }^{5)}$ found that online health information accounted for $49 \%$ of medically-based information, $1 \%$ of advertising, and $21 \%$ of mixed answers. This study also analyzed the errors in answering, which fortunately showed a continuous decrease, especially a dramatic decrease in the errors in answers related to scaling, compared to wisdom teeth or endodontic treatment. It is believed that improvements and enhancements by users for accurate information were appearing on the internet.

We could not examine more diverse dental treatments due to the large amount of online data. In addition, searching with names of treatment might reveal results inconsistent with previous studies that surveyed using names of diseases or health promotion behaviors. Despite these limitations, this study showed how online dental health information has been changing over time and its meaningfulness for generating data that could be used for delivering accurate online dental health information.

\section{Notes}

\section{Conflict of interest}

No potential conflict of interest relevant to this article was reported.

\section{Ethical approval}

This study did not contain any sensitive personal information and has not been reviewed for research ethics because it was a collection of online data.

\section{ORCID}

A-Reum Kang, https://orcid.org/0000-0003-4356-2401

Ye-Eun Go, https://orcid.org/0000-0002-3149-9428

Ka-Eun Kim, https://orcid.org/0000-0003-4849-5869

Min-Joo Kim, https://orcid.org/0000-0002-6369-5060

Seon-Jeong Kim, https://orcid.org/0000-0003-3433-2807

SooJeong Hwang, https://orcid.org/0000-0003-4725-1512

\section{References}

1. Jeong SH, Kim JG, Kim TY, Park SY, Lee SY: Survey on the consumer preference for the internet health information of the patients' online community members. J Korean Soc Med Inform 13: 207-220, 2007.

https://doi.org/10.4258/jksmi.2007.13.3.207

2. Kim SJ: An exploratory study of undergraduate students' health information needs and seeking behaviors in social media. J Korean Biblia Soc Libr Inf Sci 23: 239-260, 2012.

3. Silence E, Briggs P, Harris PR, Fishwick L: How do patients evaluate and make use of online health information? Soc Sci Med 64: 1853-1862, 2007. https://doi.org/10.1016/j.socscimed.2007.01.012

4. Choi JM: Medical/Health social network service trends. JKIITM 10: 99-106, 2012.

5. Kim TY, Kim YS, Choi SH, Kim DH, Chang YJ: Development of classification model for healthcare contents on the online community. J Inf Syst 26: 285-301, 2017. https://doi.org/10.5859/KAIS.2017.26.4.285

6. Kim MJ, Yang HJ: Research on internet counselling for oral health. Korean J Health Serv Manag 7: 251-260, 2013.

7. Oh SH, Shin NR, Hwang SJ: Analysis of online questions about dental hygienist: case report in naver intelligent network. Oral Biol Res 42: 62-66, 2018. https://doi.org/10.21851/obr.42.01.201803.62

8. Hwang SJ, Lee SM, Moon HJ, et al.: Searching information on online questions by Korean dental hygienists: case report. J Korean Acad Dent Admin 6: 43-47, 2018.

9. Ahn KS, You JY: A study on dental treatment for urban dental service users and their satisfaction level. KyungBok Nonchong 6: 485-502, 2002.

10. Heo YM, Kim HJ, Kim MN, et al.: Affecting factors on national health insurance coverage dental scaling experience. J Dent Hyg Sci 15: 287-294, 2015. https://doi.org/10.17135/jdhs.2015.15.3.287

11. Hwang YJ, Cho YS, Lee SY: Awareness and satisfaction of health insurance coverage of dental scaling. J Dent Hyg Sci 15: 620-627, 2015. https://doi.org/10.17135/jdhs.2015.15.5.620

12. Lee KH, Kim HS, Gu JH, et al.: A study on the perception of wisdom tooth extraction. J Korean Soc Dent Hyg 17: 235-245, 2017. 
https://doi.org/10.13065/jksdh.2017.17.02.235

13. Kim YK, Lim BY: Internet health counseling for Korean medicine in the NAVER Jisik-iN. J Orient Med Soc 17: 51-63, 2013.

14. Khechine H, Pascot D, Premont P: Use of health-related information from the internet by english-speaking patients. Health Inform J 14: 17-28. 2008. https://doi.org/10.1177/1460458207086331

15. Kim JE, Kim SW, Shin YJ: Survey on the physicians' views of the effects of internet health information on the doctor-patient relationship. J Cybercommun Acad Soc 21: 279-301, 2007.

16. Lee BK, Byoun WJ, Lim JL: The influence of individual's E-health literacy on doctor-patient communication. J
Cybercommun Acad Soc 27: 89-125, 2010.

17. Kim SM: A study on health information use on cues to action, self-efficacy, subjective norm, behavior intention through internet. J Sport Leis Stud 34: 1605-1614, 2008.

18. Murray E, Lo B, Pollack L, et al.: The impact of health information on the internet on health care and the physicianpatient relationship: national U.S. survey among 1.050 U.S. physicians. J Med Internet Res 5: e17, 2003 https://doi.org/10.2196/jmir.5.3.e17

19. Suh MK: Legal measures for handling internet health information. Korean J Health Educ Promot 20: 61-76, 2003.

20. Kim GS, Kim YR, Ryu SW: Health behavior stage and usage of internet health information. J Korea Soc Health Inf Stat 35: 177-193, 2010. 